\title{
Kernos
}

Revue internationale et pluridisciplinaire de religion grecque antique

$21 \mid 2008$

Varia

\section{Revue des Revues}

Vinciane Pirenne-Delforge et Angel Ruiz Pérez

Édition électronique

URL : https://journals.openedition.org/kernos/1718

DOI : 10.4000/kernos. 1718

ISSN : 2034-7871

\section{Éditeur}

Centre international d'étude de la religion grecque antique

\section{Édition imprimée}

Date de publication : 1 janvier 2008

Pagination : 373-382

ISSN : 0776-3824

\section{Référence électronique}

Vinciane Pirenne-Delforge et Angel Ruiz Pérez, «Revue des Revues », Kernos [En ligne], 21 | 2008, mis en ligne le 09 septembre 2011, consulté le 24 août 2022. URL : http://journals.openedition.org/kernos/ 1718 ; DOI : https://doi.org/10.4000/kernos. 1718 
Kernos 21 (2008), p. 373-382.

\title{
Revue des Revues
}

\author{
par Vinciane PIRENNE-DELFORGE et Angel RUIZ PÉREZ
}

Cette chronique a été réalisée à Liège par Vinciane Pirenne-Delforge et à Santiago de Compostella par Angel Ruiz Pérez, pour les revues espagnoles. Toutes les références glanées au cours du dépouillement sont également mentionnées, sans que tous les articles en question aient pu être consultés, afin de fournir un panorama le plus complet possible. Ce dépouillement concerne essentiellement les publications des années 2005 à 2007/8, auxquelles on a ajouté les tomes des revues portant un millésime antérieur mais seulement disponibles depuis peu. Nous remercions tous ceux qui pensent à nous envoyer leurs contributions sur la religion grecque.

Alekniené Tatjana, «Les 'dieux et démons' dans le Traité 1 (c. 7, 19-20) de Plotin», Hermes 135 (2007), p. 483-498 [la rencontre avec les formes ( $\varepsilon i \delta \eta)$ des dieux ou des démons évoquée par Plotin juste avant d'évoquer les images platoniciennes pourrait être une référence aux épiphanies divines de l'épopée homérique, et plus particulièrement de celles qui concernent Ulysse].

ASMIS Elizabeth, " Myth and philosophy in Cleanthes’ Hymn to Zeus », GRBS 47 (2007), p. 413-429.

BEES Robert, «Der Universalhistoriker als Diener der göttlichen Vorsehung: zu Diodor I 1, $3=$ Poseidonios, Fr. 80 Theiler », SCO 48 (2002) [2007], p. 207-232.

BELAYCHE Nicole, «Les dieux 'nomothètes'. Oracles et prescriptions religieuses à l'époque romaine impériale », RHR 224 (2007), p. 171-191 [l'existence d'oracles «spéculatifs » ne doit pas occulter le fait que les sanctuaires oraculaires à époque tardive ont continué d'être des lieux de la régulation religieuse car les considérations théologiques ne s'opposent pas au soin des rites].

BELAYCHE Nicole, RÜPKE Jörg, «Divination et révélation dans les mondes grec et romain. Présentation », RHR 224 (2007), p. 139-147.

BELLANDi Franco, «La pena di morte per l'assassinio’ del bue aratore », Philologus 151 (2007), p. 105114 [un passage difficile de Varron, partiellement corrigé et commenté par Columelle, évoque le problème posé par la mise à mort des animaux de labour; l'analyse des points de vue contradictoires se referme sur une comparaison avec les Bouphonies attiques].

Beneitez PRUDENCiO José Javier, « Nominadores bárbaros y el nombre de los dioses: una glosa al Crátilo de Platón », Ilu 12 (2007), p. 29-53 [en tenant compte des recherches d'Hérodote en matière de noms dans son récit sur l'Égypte, les jeux platoniciens du Cratyle révèlent leurs implications théologiques].

BÉrArd Claude, «'On n’y voit rien'. Du bon usage des erreurs », Pallas 75 (2007), p. 263-274 [vibrant plaidoyer pour une lecture 'objectivée' des images qui évite les identifications sans argumentation, mais sur la seule base d'un argument d'autorité...].

Berman Daniel W., "Dirce at Thebes », G \& R 54 (2007), p. 18-39 [les traditions qui se sont développées autour du lieu et de la figure de Dircè associent le nom à un lieu spécifique, mais aussi à des narrations qui vont au-delà de Thèbes et alimentent la koinè culturelle des mythes grecs].

BERNABÉ Alberto, "The Derveni theogony: many questions and some answers », HSPh 103 (2007), p. 99-133 [analyse littéraire, religieuse et philosophique du poème reconstitué dans un article publié dans Kernos 15, 2002, p. 91-129]. 


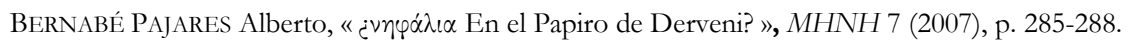

BEZZA Giuseppe, «Alcune note sull'eutocia e la distocia », MHNH 7 (2007), p. 289-292.

BILES Zachary, «Celebrating poetic victory: representations of epinika in Classical Athens », JHS 127 (2007), p. 19-37 [mise en évidence de la célébration de la victoire lors des compétitions dramatiques à Athènes; bien que mal attesté, cet événement s'inscrit dans le cadre du culte à Dionysos, qui est au cœur des célébrations dramatiques].

BONNECHERE Pierre, «Notes trophoniaques II : la dixième épigramme de Callimaque. Timarque de Chéronée (Plutarque, De genio Socratis, 589f-592f) et les croyances sur l'Hadès?», Hermes 136 (2008), p. 153-166 [mise en évidence des jeux subtils de cette épigramme autour de la descente dans l'antre de Trophonios et d'un Timarque qui pourrait s'identifier au Timarque mis en scène par Plutarque dans ce contexte trophoniaque de quête d'une vérité sur l'au-delà].

Brelaz Cédric, "L’archonte stéphanéphore et la Tyché de Lébadée», Tyche 21 (2006), p. 11-28 [dédicace du Irer s. ap. J.-C. d'une statue de Tyché par le magistrat et son épouse].

Bremer Jan Maarten, «Traces of the Hymn in the epinikion », Mnemosyne 61 (2008), p. 1-17 [les dieux sont à l'avant-plan de l'hommage chanté par les épinicies, les athlètes étant maintenus à leur place de mortel, loin du bonheur éternel des dieux immortels].

Brocato Filippo, «I santuari di Atena in Sicilia e Magna Grecia », "O@uos. Quaderni di Storia Antica 6-7 (2004-2005), p. 89-102.

BRU Hadrien, DEMIRER Ünal, « Dionysisme, culte impérial et vie civique à Antioche de Pisidie », REA 109 (2007), p. 27-49 [retour sur la politique religieuse des Antonins et des Sévères, relayée par les élites impliquées dans leur culte et dans celui de Dionysos-Liber en Asie Mineure].

BYL Simon, «Les scholies des Nuées d'Aristophane et l'initiation éleusienne », BAGB (2008), p. 96-100.

CABANES Pierre, «Thétis et Achille à Apollonia d'Illyrie », REA 109 (2007), p. 529-540 [deux bases de statues portant dédicace (déb. hellénistique) témoignent d'un culte rendu au héros achéen et à sa mère dans la cité qui a choisi Apollon, protecteur des Troyens, comme fondateur].

Cabrera Paloma, Bernabé Alberto, "Échos littéraires de l'enlèvement de Perséphone. Un vase apulien du Musée Archéologique National de Madrid », $A K 50$ (2007), p. 58-75 ['’imagerie de ce loutrophore représentant notamment l'enlèvement de Perséphone pourrait avoir été influencée par des versions orphiques du mythe, le vase évoquant le cycle de la vie et de la mort].

CALVo MARTíneZ José Luis, «Magos griegos o persas: los usos más antiguos del término magos, Heráclito, Sófocles, Eurípides y el Papiro de Derveni », MHNH 7 (2007), p. 301-314.

CAMPANELli Sara, "Kline e synklitai nel culto di Hypsistos: nota su due iscrizioni del Serapeo di Tessalonica », ZPE 160 (2007), p. 123-133.

CARASTRO Marcello, "Quand Tirésias devint un mágos. Divination et magie en Grèce ancienne (V_ IVe siècle av.n.è.) ", RHR 224 (2007), p. 211-230 [en tenant compte des catégories antiques ellesmêmes, on constate que les Grecs ont conçu la notion de magie dans un contexte de polémiques contre le savoir traditionnel, au $\mathrm{V}^{\mathrm{e}} \mathrm{s}$. av.n.è. et que les connotations négatives ont servi à stigmatiser les devins, disqualifier leurs pratiques et dénoncer leur influence politique].

CARruesco Jesús, «La rame et l'aiguillon. Les morts d'Ulysse », Mètis n.s. 4 (2006), p. 123-137 [les différentes versions de la mort d'Ulysse renvoient toutes au champ sémantique de la navigation et de la fondation].

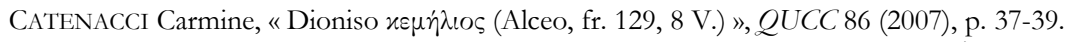

Christopoulos Menelaos, "Quelques remarques sur Hélène dans l'Odyssée. À la recherche des innovations mythographiques et narratives », Gaia 11 (2007), p. 101-120.

CRAWLEY QUINN Josephine, « Herms, kouroi and the political anatomy of Athens », G \& R 54 (2007), p. 82-105 [dans le cadre des expérimentations "démocratiques" à la fin du VIe s., les piliers hermaïques ont fait une apparition aggressive dans la culture visuelle de l'Attique, en créant un contraste avec l'image des kouroi aristocratiques et en interagissant avec elle].

CRISTILLI Armando, «L'Iside Pelagia di Budapest: problemi di iconografia e di cronologia », BABesch 82 (2007), p. 201-206.

Cusumano Nicola, « Polivalenze funzionali e figurative. Osservazioni su Zeus Meilichios », Mètis n.s. 4 (2006), p. 165-192 [analyse des fonctions du dieu, notamment celle qui consiste à reconstituer le tissu politique et social continûment exposé à des déchirures, et de la pluralité de ses figurations]. 
DAIX David-Arthur, «Réalités et vérités dans la Théogonie et les Travaux et les Jours d’Hésiode », Mètis n.s. 4 (2006), p. 139-164 [divine dans la Théogonie, humaine dans Les Travaux et les Jours, la vérité dans l'œuvre d'Hésiode offre deux visages, selon qu'elle habite le temps des dieux ou celui des hommes]

DARBO-PESCHANSKI Catherine, « La folie pour un regard. Oreste et les divinités de l'échange (Érinyes, Euménides, Charites) », Mètis n.s. 4 (2006), p. 13-28.

DAVIES Malcolm, «Triton and the Argonauts in Pindar and Apollonius », SCO 48 (2002) [2007], p. 5368.

DESPINIS Giorgos I., «Iphigeneia und Orestes. Vorschläge zur Interpretation zweier Skulpturen funde aus Brauron », $M D A I(A) 120$ (2005), p. 241-267.

Di Nino Margherita Maria, « Asclepius' cult at the court of the Ptolemies », Hermes 136 (2008), p. 167 187 [analyse des iamata conservés parmi les épigrammes du « nouveau » Posidippe].

DíEz de Velasco ABellán Francisco P., «El mito que mira a la muerte », Gerión Extra 1 (2007), p. 231-234.

DILLON Matthew, «Were Spartan women who died in childbirth honoured with grave inscriptions?

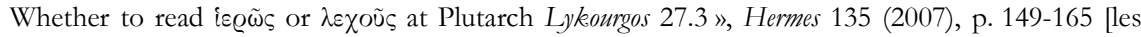
femmes de Sparte qui mouraient au moment où elles occupaient une charge religieuse se voyaient octroyer une stèle funéraire inscrite et non les femmes qui mouraient en couches].

DuRBEC Yannik, «Les avatars d'une formule indo-européenne : de la mort des dragons et de quelques autres monstres chez les poètes hellénistiques », PP 348 (2006), p. 161-175.

ELLINGER Pierre, "Artémis, l'histoire et les historiens », Europe. Revue littéraire mensuelle 945-946 (2008), p. 122-146 [mise en perspective du rôle d'Artémis dans l'écriture de l'histoire par les Anciens et de la «théologie » de la déesse telle qu'elle est utilisée comme grille d'explication traditionnelle].

EMONDS Radcliffe G., III, «Extra-ordinary people: mystai and magoi, magicians and Orphics in the Derveni papyrus », CPh 103 (2008), p. 16-39 [la construction moderne des catégories de «magie» et d'« orphisme » explique pourquoi il fut si difficile de classer l'auteur du papyrus; en prenant ces processus en considération et en retraçant les perspectives internes à la culture grecque, on comprend mieux la manière dont les Grecs élaboraient leurs idées sur la religion].

FAULKNER Andrew, "The legacy of Aphrodite: Anchises' offspring in the Homeric Hymn to Aphrodite », AJPh 129 (2008), p. 1-18 [discussion de l'interprétation qui voit dans l'hymne la description de la limitation des pouvoirs d'Aphrodite et la fin de l'union des immortels et des mortels; remise à l'honneur de l'interprétation qui associe l'hymne à des Aineiades au pouvoir en Troade].

Ferrari Franco, Prauscello Lucia, «Demeter Chthonia and the Mountain Mother in a new gold tablet from Magoula Mati », ZPE 162 (2007), p. 193-202 [contre l'avis des éditeurs de la lamelle (Parker-Stamatopoulou, cf. infra), le texte est rapporté à l'arrière-plan "orphico-dionysiaque » des autres lamelles].

FEYEL Christophe, «La dokimasia des animaux sacrifiés », RPh 80 (2006), p. 35-58 [une telle inspection permettait de s'assurer que l'animal serait agréé par la divinité, mais aussi qu’il serait effectivement sacrifié].

Fialho Maria do Céu, «O deus de Delfos na Electra de Sófocles », Minerva 20 (2007), p. 39-52.

FLETCHER Judith, « A trickster's oaths in the Homeric Hymn to Hermes », AJPh 129 (2008), p. 19-46 [le motif du serment structure l'hymne et le passage d'Hermès à l'âge adulte, avec la confirmation de ses timai; la sophistication de l'hymne est une offrande adaptée à l'Hermès des tours et détours].

FLETCHER K.F.B., «Systematic genealogies in Apollodorus’ Bibliotheca and the Exclusion of Rome from Greek myth », CAnt 27 (2008), p. 59-91 [il est nécessaire d'envisager le projet de la Bibliothèque comme un tout, qui relie la Grèce à d'autres lieux et d'autres peuples, surtout au Proche-Orient; l'exclusion de Rome signifie qu'elle n'a pas de rapport avec la Grèce et est de peu d'importance...].

FrONING Heide, «Überlegungen zur Aphrodite Urania des Phidias in Elis », MDAI(A) 120 (2005), p. 285-294 [une terre cuite d'Élis représente un pied chaussé d'une sandale posé sur une tortue, renvoyant à l'Aphrodite Ourania sculptée par Phidias pour la cité, comme pour Athènes].

Frontisi-Ducroux Françoise, «L'étoffe des spectres", Mètis n.s. 4 (2006), p. 29-50 [analyse des procédures verbales et visuelles pour donner à voir l'invisible, tant dans la manière dont Eschyle porte les Érinyes à la scène que dans la Bouclier d'Hésiode où Achlus incarne l'abolition de la vision]. 
GAGNÉ Renaud, «Winds and ancestors: the Physika of Orpheus », HSPh 103 (2007), p. 1-23 [un traité orphique des Physika, remontant au Ve s. av. J.-C., pourrait avoir été à l'origine de la tradition littéraire autour de la figure des Tritopatores qui, d'ancêtres communs à un groupe, sont devenus les ancêtres de l'humanité].

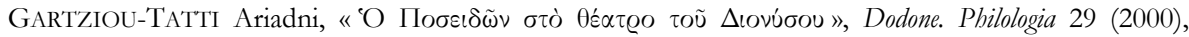
p. 237-364.

GAWLINSKI Laura, "The Athenian calendar of sacrifices. A new fragment from the Athenian Agora », Hesperia 76 (2007), p. 37-55 [editio princeps d'un nouveau fragment très mutilé : on y trouve notam-

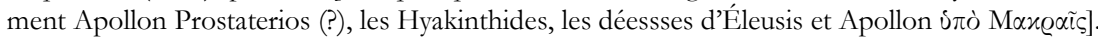

GHERCHANOC Florence, «Le(s) voile(s) de l'échange dans le monde grec: se voiler, se dévoiler. La question particulière des anakaluptêria», Mètis n.s. 4 (2006), p. 239-267 [exploration de la nature, des fonctions et des valeurs du voile de la numphê et des anakaluptêria à l'intérieur du rituel matrimonial].

Gosserez Laurence, «Le Phénix coloré d'Hérodote à Ambroise de Milan », BAGB (2007), p. 94-117.

GRAF Fritz, "The oracle and the image. Returning to some oracles from Claros », ZPE 160 (2007), p. 113-119 ['importance numérique des sentences oraculaires clariennes autour de statues est remarquable et montre que ces objets devaient résoudre une crise traversée par les consultants, en référence à d'antiques pratiques rituelles présumées].

GRAF Fritz, "Untimely death, witchcraft, and divine vengeance. A reasoned epigraphical catalog », ZPE 162 (2007), p. 139-150 [catalogue des inscriptions qui sollicitent une vengeance divine à la suite de la mort suspecte d'une personne trop tôt disparue, auquel s'ajoutent des épitaphes non explicites accompagnées du symbole de deux bras levés, paumes ouvertes].

GRANGER Herbert, "The theologian Pherecydes of Syros and the early days of natural philosophy ", HSPh 103 (2007), p. 135-163 [le traité de Phérécyde «Sur la nature et les dieux » atteste que la théologie révolutionnaire d'Anaximandre est intervenue dans un mouvement plus ample, dont le détail nous échappe].

GRANINGER Denver, «Studies in the cult of Artemis Throsia », ZPE 162 (2007), p. 151-164 [remise en cause de l'interprétation initiatique des dédicaces thessaliennes d'Artémis Throsia proposée en dernier lieu par M.B. Hatzpoulos, Cultes et rites de passage en Macédoine (1994)].

HAACK Marie-Laurence, «Apollon médecin en Étrurie », AncSoc 37 (2007), p. 167-190 [l’abondance des ex-voto anatomiques dans les dépôts votifs hellénistique pourrait trouver sa justification dans la popularité du culte d'Apollon].

HAACK Marie-Laurence, «Phocéens et Samiens à Gravisca », BABesch 82 (2007), p. $29-40$ [contestation de l'idée que l'emporion serait une fondation phocéenne autour d'un sanctuaire d'Aphrodite au profit de l'implantation samienne d'une escale en ce lieu, avec un sanctuaire d'Héra].

Halm Tisserant Monique, "Symbolique et éthique du blanc et du noir dans la pensée et dans l'imagerie en Grèce ancienne », Eidola 3 (2006), p. 9-28 [place respective des valeurs du noir et du blanc - davantage en relation avec la luminosité que la couleur - dans les perceptions visuelles grecques et dans la représentation du monde].

HAME Kerri J., «Female control of funeral rites in Greek tragedy: Klytaimestra, Medea, and Antigone », CPh 103 (2008), p. 1-15 [le message concernant les attitudes adéquates dans le cadre des funérailles tel que le véhicule la tragédie s’applique à la fois aux hommes et aux femmes, pour peu que l'on replace ces drames sur l'arrière-plan des pratiques historiques].

Hamon Patrice, «Un prêtre des dieux boulaioi dans le bâtiment du Conseil de Cos (I. Cos ED 32)», Chiron 36 (2006), p. 151-169 [l'inscription en question témoigne de l'existence d'un "prêtre du bouleutèrion », ce qui atteste la tendance des cités grecques à transformer peu à peu la salle du conseil en véritable sanctuaire, comme l'était déjà le prytanée].

HeIDEN Bruce, «The Muses’ uncanny lies: Hesiod, Theogony 27 and its translators », AJPh 128 (2007),

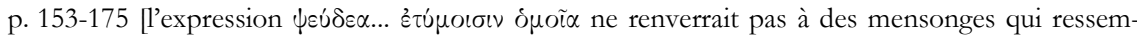
blent à des réalités, mais à des mensonges qui équivalent à la vérité; il y aurait donc une même revendication de vérité au vers 27 qu'au vers 28 qui évoque les $\alpha \lambda \lambda \eta \dot{c} \alpha]$.

HöLBL Günther, «Die Aegyptiaca von Aphroditetempel auf Thera », MDAI(A) 121 (2006), p. 73-103 [publication des objets égyptiens mis au jour dans ce sanctuaire et qui appartiennent à la $1^{\text {re }}$ phase 
du sanctuaire $\left(\mathrm{VIII}^{\mathrm{e}} / \mathrm{VII}^{\mathrm{e}} \mathrm{s}\right.$.); une partie de ce matériel est proche des découvertes faites à Érétrie, ce qui atteste que des Eubéens - et pas seulement des Phéniciens - ont pris part à la diffusion].

HuysCOM-HAXHi Stéphanie, Muller Arthur, «Déesses et/ou mortelles dans la plastique de terre cuite. Réponses actuelles à une question ancienne», Pallas 75 (2007), p. 231-247 [chronique bibliographique]

IBÁÑEZ CHACÓN Álvaro, «Un posible fragmento inédito de la Heraclea de Conón en la Mitología de Natale Conti », Myrtia 22 (2007), p. 83-93.

IVERSEN Paul A., "The small and great Daidala in Boiotian history », Historia 56 (2007), p. 381-418 [parcours et analyse des mentions de l'Héra de Platées à tous les moments clés de l'histoire de la cité; étude de la périodicité des Daidala].

JAILlARD Dominique, "Kûdos arésthai (emporter le kêudos). Le kûdos des rois, des guerriers et des athlètes au miroir des dieux », Gaia 11 (2007), p. 85-99.

JAILlARD Dominique, «Plutarque et la divination : la piété d'un prêtre philosophe », RHR 224 (2007), p. 149-169 [Plutarque réunit en sa personne le praticien public et l'exégète, et en réfléchissant sur les enjeux du rite, le philosophe accomplit la piété ancestrale].

JOURDAIN-ANNEQUIN Colette, "Héraclès chez Syleus ou le héros entre soumission et résistance », SHHA 25 (2007), p. 147-161

KASSEL Rudolf, «Das Opfer des Stratonikos », ZPE 163 (2007), p. 44 [concerne la part de la prêtresse lors d'un sacrifice à une Aphrodite chypriote dans Gnomologicum Vaticanum, 527 Sternbach].

KNIGGE Ursula, «Ein Grabmonument der Alkmeoniden im Kerameikos », MDAI(A) 121 (2006), p. 127-163 [le jeune homme représenté pour être l'olympionice Alkméon, commémoré avec son fils Mégaklès dans un autre monument funéraire; deux tombes sous un tumulus pourraient leur appartenir, en relation avec le sanctuaire des Tritopatreis, leur servant de lieu de culte familial].

KOnSTAN David, "Medea: a hint of divinity?", CW 101 (2007), p. 93-94 [le début de la Médée d'Euripide anticipe sur le statut quasi divin qu'elle assume à la fin].

Kourayos Yannos, BuRns Bryan, "Exploration du sanctuaire archaïque à Mandra sur l'île de Despotiko », BCH 128-129 (2004-2005), p. 133-174 [identification de cinq pièces parallèles qui pourraient être un hestiatorion; mise au jour de nombreux objets votifs et de mobilier archaïque; les pièces architecturales attestent la présence de plusieurs bâtiments doriques; le nom d'Apollon sur des tessons invite à lui attribuer le sanctuaire, où Artémis semble avoir été également vénérée].

KOUSSER Rachel, « Mythological group portraits in Antonine Rome: The performance of myth », $A J A$ 111 (2007), p. 673-692 [les sculptures juxtaposent des corps divinement idéalisés sur le modèle d'Arès et Aphrodite avec des portraits réalistes, offrant dès lors un moyen de représenter les qualités affectives du mariage romain par une référence au mythe et à l'art grecs].

KUNST Christiane, "Frauen im hellenistischen Herrscherkult», Klio 89 (2007), p. 24-38 [le culte des femmes des dynasties hellénistiques est étroitement lié à la place de leur mari; l'identification à Aphrodite est largement favorisée, parce qu'elle correspond aux différents aspects de l'existence féminine et permet de concevoir les différents aspects de l'influence des femmes sur les hommes].

LARSON Stephanie, "Reassessing an archaic Boiotian dedication (Delphi Museum Inv. no. 3078)», ZPE 162 (2007), p. 99-107 [inscription de la $2^{\mathrm{e}}$ moitié du VI $\mathrm{I}^{\mathrm{e}}$ s. qui doit avoir associé Apollon et Athéna sous l'appellation de Tritogeneia dans la dédicace d'une figurine en bronze de la part des Béotiens].

LEHOUX Daryn, "Drugs and the Delphic oracle », $C W 101$ (2007), p. $41-56$ [examen critique des études qui associent l'inspiration delphique à une intoxication au gaz, avant de réévaluer la documentation sur laquelle une telle interprétation est fondée].

LENZI Mary, " Plato’s last look at the gods: philosophical theology in law », Philotheos 7 (2007), p. $124-$ 135.

LEVANIOUK Olga, «The toys of Dionysos », HSPh 103 (2007), p. 165-202.

LEVENTI Iphigeneia, "The Mondragone relief revisited. Eleusinian cult iconography in Campania », Hesperia 76 (2007), p. 107-141 [le relief renvoie à des épisodes de l'Hymne hom. à Déméter; la figure de Dionysos sur le trône d'Hadès indique que ce relief attique avait été dédié dans un sanctuaire campanien par des initiés éleusiniens ayant aussi participé à des mystères orphico-dionysiaques]. 
LiAPIS Vayos J., «Zeus, Rhesus, and the Mysteries», CQ 57 (2007), p. 381-411 [le Rhésos fournit une étiologie pour un culte thrace qui met en relief l'association du héros avec Zeus dans le cadre de mystères; la pièce pourrait avoir été écrite pour une représentation à la cour de Macédoine].

LissarRague François, «Comment peindre les Érinyes ?», Mètis n.s. 4 (2006), p. 51-70 [examen des éléments mis en œuvre pour rendre visible les figures d'invisibilité que sont les Érinyes].

Lougovaya-Ast Julia, " Myrrhine, the first priestess of Athena Nike », Phoenix 60 (2006), p. 211-225 [l'épitaphe de Myrrhine ferait référence à deux moments différents: sa désignation comme prêtresse et l'association de sa charge au nouveau temple d'Athéna, ce qui résoud les problèmes de chronologie des autres inscriptions de ce dossier; annexe sur la datation de l'Athéna Promachos].

LOZANO Velilla Arminda, "Devoción religiosa y sumisión personal a la divinidad en las aldeas minorasiáticas lidio-frigias », SHHA 25 (2007), p. 341-355

LOZANO Velilla Arminda, «La confesión de los pecados, expresión de la sumisión a la divinidad », Gerión Extra 1 (2007), p. 235-242

LUQUe MORENO Jesús, « Agua de Éstige, agua de la vida », MHNH 7 (2007), p. 147-182 [évolution de la représentation des eaux du Styx qui, de synonyme des enfers, ont été transportées au ciel dans une nouvelle perspective eschatologique qui identifie le terme à la vie].

LUQUE MORENO Jesús, « Agua de Éstige, agua del horror », Florentia Iliberritana 18 (2007), p. 251-309.

LYTLE Ephraim, «Fishless mysteries or high prices at Athens? Re-examining IG II $^{2} 1103$ », MH 64 (2007), p. 100-101 [la lettre d'Hadrien que reproduit cette inscription fragmentaire entend réguler les prix à Athènes et rencontre des préoccupations civiques et non religieuses].

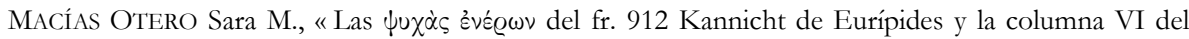
Papiro de Derveni », $C F C(G) 17$ (2007), p. 145-162 [ce fragment présente une vision du caractère intermédiaire des âmes qui entre en résonance avec les conceptions orphiques et est complémentaire du papyrus de Dervéni en relation avec la présence des daimones dans le rituel orphique].

MACías ViLLALOBOS Cristóbal «Los cometas en el mundo antiguo: entre la ciencia y la superstición », Veleia 23 (2006), p. 41-71

MACKINNON Michael, "Osteological research in Classical archaeology », AJA 111 (2007), p. 473-504 [cette mise au point d'un anthropologue s'assortit d'une remarquable bibliographie en ligne: http://www.ajaonline.org/pdfs/111.3/AJA1113_MacKinnon_BIB.pdf].

MACRIS Constantinos, «Becoming divine by imitating Pythagoras?», Mètis n.s. 4 (2006), p. 297-329 [analyse de la relation entretenue par la figure d'Apollonius de Tyane et celle d'Alexandre d'Abonoutichos avec le référent par excellence en terme d'« homme divin» qu'était Pythagore].

Mangou Helen, Petropoulos Michalis, Gasparatos Alexander, Tsakmakis Elias, IoAnnou Panayiotis V., "The temple of Artemis (F)aontia, at Rakita, Achaia, Greece: chemical composition of metal and glass votives ", OAth 31-32 (2006-2007), p. 211-220.

MARCH Duane A., «Kleisthenes and the league of Athena Pallenis », Historia 57 (2008), p. 134-141 [la ligue d'Athéna Pallènis pourrait avoir joué un rôle lors du coup de force de Cléomène, ce qui explique que le groupement des dèmes par Clisthène ait cassé les solidarités régionales de la ligue dans la composition des trittyes].

MARLIER Thomas, «Pausanias archéologue : le cas des statues», REA 108 (2006), p. 433-451 [dans l'évaluation scientifique des statues, Pausanias effectuerait un certain nombre d'opérations scientifiques qui peuvent être comparées à celles pratiquées par les archéologues modernes].

Mendez Dosuna Julián, «Le skyphos de Satyros et le kelês de Dorilaos : une consultation oraculaire de Dodone (Lhôte n 113) », ZPE 162 (2007), p. 181-187.

MendeZ DosunA Julián, « Notes de lecture sur les lamelles oraculaires de Dodone », ZPE 161 (2007), p. 137-144 [corrections de certaines restitutions proposées par É. Lhôte dans son édition des lamelles].

MitChell-BOyaSK Robin, "The Athenian Asklepieion and the end of Pbiloctetes», TAPhA 137 (2007), p. 85-117 [examen des relations existant entre la fin du drame et le cadre athénien de la performance, avec notamment le tout nouveau sanctuaire d'Asclépios construit au flanc sud de l'acropole]. 
MONBRUn Philippe, «La chouette-hoplite d'Athéna et la Crète des archers. Les «dessous » d'une allergie réciproque », REA 109 (2007), p. 559-581 [l'oiseau d'Athéna, tout entier du côté de la lance et du bouclier, ne peut vivre dans la Crète de l'arc et des flèches; il s'agit d'une traduction éthologique de l'opposition entre hoplite et archer].

MOORE Mary B., «Athens 803 and the ekphora », AK 50 (2007), p. 9-23.

NADAL Éléonore, «De la cavalerie à la flotte athénienne: l'iconographie attique de Poséidon et l'histoire d'Athènes », Pallas 75 (2007), p. 151-168 [entre VI et IV $\mathrm{V}^{\mathrm{e}} \mathrm{s}$. , l'iconographie du dieu montre une évolution qui le fait passer de la fonction de maitre des chevaux à celle de divinité marine, à laquelle sont associées les orientations « démocratiques » de son fils supposé, Thésée].

NAIDEN F.S., "The fallacy of the willing victim», JHS 127 (2007), p. 61-73 [remise en cause de l'hypothque qu'un assentiment était attendu de la part de l'animal sacrificiel avant sa mise à mort; l'agitation en question, faisant suite à l'aspersion de liquide, fait en réalité référence à la vitalité attendue de l'animal, attestant qu'elle est adéquate pour le dieu destinataire].

O’BryHim Shawn, "Adonis in Plautus' Pseudolus», CPh 102 (2007), p. 304-307 [les allusions au mythe et au culte d'Adonis au début de la pièce trouvent leur origine dans le modèle grec de Plaute].

OAKLEY John H., "The departure of the Argonauts on the Dinos painter's bell krater in Gela ", Hesperia 76 (2007), p. 347-357 [l'image ne représente pas le départ de Thésée pour la Crète, mais le départ des Argonautes de Colchide, avec une représentation d'Apsyrtos et d'Idyia].

PÀmIAS I MASSANA Jordi, «Les genealogies de Ferecides d'Atenes: entre raó i mite », Faventia 28 (2006), p. 29-35

PAPAGEORGIOU Irini, «Eine reitende Kourotrophos-Göttin geometrischer Zeit im Benaki-Museum », $M D A I(A) 120$ (2005), p. 1-31 [l'image de l'enfant, d'un âge déjà avancé, souligne davantage le caractère protecteur de l'image que le côté « maternel» de la figure féminine; l'idée ancienne de la « fertilité » semble ici combinée au nouvel idéal de la classe aristocratique; on ignore la provenance de la pièce, dont la fabrication pourrait être argienne ou corinthienne].

Papazarkadas Nikolaos, Thonemann Peter, "Athens and Kydonia: Agora I 7602 », Hesperia 77 (2008), p. 73-87 ['article aborde notamment la question des relations diplomatiques fondées sur la

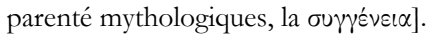

PARADISO Annalisa, "Ravir des fromages à l'autel d’Orthia ?", Ktèma 32 (2007), p. $310-325$ [la République des Lacédémoniens, 2, 9, de Xénophon décrit le rituel du vol, par de jeunes gens, de

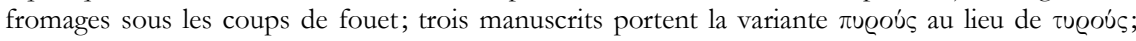
examen des liens d'Artémis avec le fromage, d'une part, avec le blé, de l'autre].

PEREA YÉBENES Sabino, «Santuario-hospital de Asclepio en Pérgamo: noticia de Rufo de Éfeso, en Oribasio », MHNH 7 (2007), p. 199-216

PerotTi Pier Angelo, « Per una definizione di Zeus-Luce: spunti esiodei », Minerva 20 (2007), p. 23-37

PERRIN-SAMINADAYAR Éric, «L'accueil officiel des souverains et des princes à Athènes à l'époque hellénistique », BCH 128-129 (2004-2005), p. 352-375 [cet accueil comportait entre autres choses des sacrifices dans les sanctuaires publics].

PILZ Oliver, «Dionysos auf einem Tonrelief der klassischen Zeit in Berlin? », MDAI(A) 120 (2005), p. 269-283 [représentation du dieu au banquet écoutant une harpiste au pied de sa klinè; toutes les répétitions du motif pourraient remonter à un prototype commun, une peinture monumentale].

PINI Ingo, "Die Siegel und die Siegelbadrücke auf Gefasshenkeln aus dem Heiligtum von Symi », $M D A I(A) 121$ (2006), p. 1-11 [certains des sceaux et des impressions de sceaux sur de la céramique mis au jour au sanctuaire de Kato Symi étaient sans doute des offrandes votives].

QuirogA A. R., « La magia y lo sobrenatural como elementos literarios en los discursos de Libanio de Antioquía », MHNH 7 (2007), p. 269-282

REECE Steve, "Homer's asphodel meadow », GRBS 47 (2007), p. $389-400$ [l'ambiguité théologique

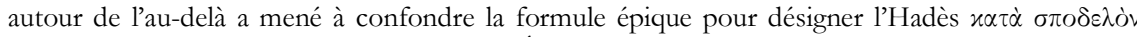

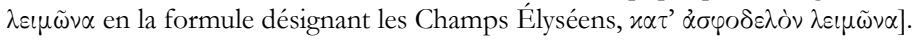

Renaut Luc, "Ptolémée Philopator et le stigmate de Dionysos », Mètis n.s. 4 (2006), p. 211-238 [l'initiation bachique n'impliquait pas de marquage corporel à la feuille de lierre, si l'on relit de manière critique les documents allégués en ce sens]. 
RicCARDi Lee Ann, "The bust-crown, the Panhellenion, and Eleusis. A new portrait from the Athenian Agora ", Hesperia 76 (2007), p. 365-390 [trouvé près de l'Eleusinion de la cité, le buste porte une couronne ornée de portraits impériaux; il pourrait s'agir d'un archonte ou d'un agonothète des Panhellenia, le Panhellénion étant étroitement associé à Éleusis].

RICCIARDELLI Gabriella, «I nomi di animali nelle tavolette di Tebe: una nuova ipotesi », PP 361 (2006), p. 241-263 [interprétation des noms d'animaux, auxquels sont offerts des aliments, comme étant des anthroponymes, en comparaison de situations ultérieures où de tels noms renvoient à des personnes occupant certaines charges dans des cultes ou dans le cadre de célébrations festives].

Rodríguez Somolinos Helena, «La planta de la inmortalidad en Grecia y el mito de Glauco de Antedón », Epos 22 (2006), p. 11-19

RODRÍGUEZ-NORIEGA GUILLÉN Lucía, «Intentando socavar una falsa creencia: la identidad del Ave de Atenea ", Studium. Revista de bumanidades 12 (2006), p. 103-111

ROOD Naomi, "Hesiod's metallurgy simile (Th. 861-7)», PCPS 53 (2007), p. 112-123 [l'image métallurgique qui clôt le combat de Zeus contre Typhon signifie la création d'un ordre par la séparation des éléments mêlés dans le chaos, en mêlant la ruse et la force].

Rosivach Vincent J., «Why seize the acropolis? », Historia 57 (2008), p. 125-133 [si Cylon, Pisistrate ou Cléomène ont inauguré leur coup d'état en prenant l'acropole, c'est parce que le prytanée se situait originellement en ce lieu].

ROSTAD Aslak, "The religious context of the Lydian propitiation inscriptions », SO 81 (2006), p. 88108 [le contenu des stèles «de confession» peut être analysé dans les mêmes catégories que les inscriptions religieuses provenant d'autres lieux d'Asie Mineure; un grand nombre de textes s'adressent aux dieux en tant que pourvoyeurs de bienfaits et non comme divinités vengeresses].

SAÏD Suzanne, «Les dons de Prométhée et leur valeur dans le 'Prométhée enchainé' à la lumière d'une comparaison avec Hésiode, Platon et Aelius Aristide », Lexis 24 (2006), p. 247-263.

SALVIAT François, «La pensée de Pindare et la 2e Olympique », JS (2007), p. 3-85, 173-259 [cet article en deux volets, qui a presque la taille d'un livre, revisite en profondeur la poésie de Pindare et y identifie une profonde influence de l'orphisme archaïque ainsi que la connaissance approfondie des " présocratiques », dont Pindare pourrait faire partie].

SANTANGELO Federico, "Prediction and divination in Diodorus», DHA 33 (2007), p. 115-126 [l'histoire étant prophétesse de vérité, pour Diodore, elle doit tenir compte de l'importance de la divination; sa vision de la providence pourrait être d'inspiration stoïcienne].

Santos Silva María de Fátima, «Um Deus em busca de identidade. Dioniso em Râs», Minerva 20 (2007), p. 53-64

SAUZEAu Pierre, «De la déesse Héra à la Panaghia. Réflexions sur le problème des continuités religieuses en Grèce et en Grande-Grèce », RHR 224 (2007), p. 289-317 [le syncrétisme entre Héra, "grande déesse totalisante», et la Vierge Marie serait largement attesté, témoignant non de prétendues «survivances » mais d'un processus continu de création religieuse].

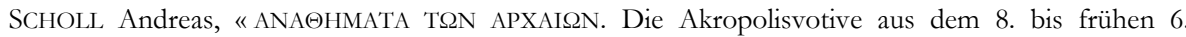
Jahrhundert v.Chr. und die Staatswerdung Athens ", JDAI 121 (2006), p. 1-173 [copieux article qui offre une importante mise au point sur les offrandes dédicatoires archaïques mises au jour sur l'acropole d'Athènes].

SChWARZMAier Agnes, «'Ich werde immer kore heissen'. Zur Grabstele der Polyxena in der Berliner Antikensammlung. Mit einem Anhang zu den Schmuckgarnituren aus der Grossen Blisniza auf der Halbinsel Taman », JDAI 121 (2006), p. 175-226 [Polyxène a été interprétée comme une prêtresse portant la statue de la divinité qu'elle servait; il s'agit plutôt d'une jeune femme morte avant le mariage et qui est représentée comme une numphè qui dédie poupée et ceinture (perdue)].

SCHWEITZER Eugen, « Plato's proof of god's existence », Pbilotheos 7 (2007), p. 136-143.

SCODEL Ruth, «The gods" visit to the Ethiopians in Iliad 1», HSPh 103 (2007), p. 83-98.

SERRATI John, "A Syracusan private altar and the development of ruler-cult in Hellenistic Sicily ", Historia 57 (2008), p. 80-91 [Syracuse, au III ${ }^{\mathrm{e}}$ s. av. J.-C., appartenait bel et bien au monde des monarchies hellénistiques et l'association de Hieron avec Zeus, qu'elle émane du roi ou de ses sujets, montre un type de relation entre un souverain et son peuple existant aussi à l'est de la Méditerranée]. 
SimANTONI-BOURNIA Eva, «Un masque humain à Hyria de Naxos, nouveau témoignage de contacts chypriotes ", BCH 128-129 (2004-2005), p. 119-132 [découverte de fragments d'un masque idéalisé barbu de la fin $\mathrm{VIII}^{\mathrm{e}}$ - début $\mathrm{VII}^{\mathrm{e}}$ s.; l'A. fait l'hypothèse qu'il était porté lors d'un rituel d'union entre Dionysos et une déesse de la fécondité identifiée à Ariane].

Sineux Pierre, "Asklépios, les Nymphes et Achéloos: réflexions sur une association cultuelle", Kentron 22 (2006), p. 177-198 [au départ d'une inscription attestant cette association à Lébéna en Crète, l'étude d'autres cultes du même type montre que, à date ancienne, les Nymphes et Achéloos sont essentiellement rapportés à la sauvegarde de la source et non à des pouvoirs guérisseurs].

SINEUX Pierre, "Une nuit à l'Asklépieion dans le Ploutos d'Aristophane : un récit dans le théâtre pour l'étude du rite de l'incubation », Mètis n.s. 4 (2006), p. 193-210.

SKEMPIS Marios, «Ery-chthonios: etymological wordplay in Callimachus Hec. fr. 70.9 H. », Hermes 136 (2008), p. 143-152 [le fragment ne nomme pas Erichthonios, mais joue sur le rapprochement des mots éQu $\mu \alpha \chi$ Өovós; analyse des étymologies du terme et de l'bapax Erychthonios].

SOMMERSTEIN Alan H., "Swearing by Hera: a deme meme? », CQ 58 (2008), p. 326-331 [l'habitude de Socrate de jurer par Héra, parallèle à ce que font d'autres personnages mis en scène par Platon, pourrait être une caractéristique du dème d'Alopèkè, où Héra avait peut-être un culte important].

SPANOUDAKIS Konstantinos, "Icarius Jesus-Christ? Dionysiac passion and biblical narrative in Nonnus' Icarius episode (Dion. 47, 1-264) », WS 120 (2007), p. 35-92 [Nonnos aurait associé les meurtrier d'Icarios aux juifs mettant le Christ à mort et Erigone à Marie-Madeleine; l'épisode est conçu comme une passion de substitution, essentielle pour l'accession de Dionysos au ciel].

STALlsmith Allaire B., "The name of Demeter Thesmophoros », GRBS 48 (2008), p. 115-131 [l'épiclèse et la fête renvoient à la fonction de la déesse qui révèle à l'humanité les rites secrets pour produire une moisson abondante, vitale pour la survie des communautés; Thesmophoros signifie dès lors « celle qui apporte l'agriculture et les rites secrets »].

STEUERNAGEL Dirk, "Griechische Tempel in der Zeit römischer Herrshaft », Das Altertum 53 (2008), p. 154-163 [analyse de l'impact contrasté de différents temples à différentes périodes : Paestum au temps de la République, Corinthe au $\mathrm{I}^{\mathrm{er}}$ s. ap. J.-C., Cyrène au $\mathrm{II}^{\mathrm{e}} \mathrm{s}$. ap. J.-C.].

STIRLING Lea M., "Pagan statuettes in late antique Corinth. Sculpture from the Panayia Domus », Hesperia 77 (2008), p. 89-161 [analyse de neuf statuettes provenant probablement d'un sanctuaire domestique : Artémis (2), Asclépios (2), Roma, Dionysos, Héraclès, Europa/Sosandra, Pan].

STRASSER Jean-Yves, «Les Olympia d'Alexandrie et le pancratiaste M. Aur. Asklèpiadès », BCH 128129 (2004-2005), p. 421-468 [concours créés sous Marc-Aurèle; mention d'un hellanodice d'Alexandrie qui a fait ériger, en 246/7, une statue de Sarapis Polieus].

STROCKA Volker Michael, «Ein klassischer Hausaltar », $A A$ (2006), p. 1-7 [l'interprétation de la pièce comme accoudoir de trône monumental est contestée en faveur d'un autel domestique attique représentant Zeus Herkeios ou Teleios entre Héra Teleia et Aphrodite Ourania].

TOPPER Kathryn, "Perseus, the maiden Medusa, and the imagery of abduction », Hesperia 76 (2007), p. 73-105 [l'apparence harmonieuse de la gorgone dans l'imagerie des vases à figures rouges provient des messages envoyés par les peintres sur la victoire de Persée; en outre, la mort de Méduse est représentée comme une perversion du schéma des enlèvements érotiques].

TUELler Michael A., "An allusive reading of the Orpheus episode in Hermesianax fr. 7 », CB 83 (2007), p. 93-108.

TURCAN Robert, «Dieux barbus du cycle bachique», JS (2007), p. 87-112 [analyse du personnage barbu qui accompagne plusieurs scènes bachiques d'époque impériale : il pourrait s'agir de formes différentes de Dionysos, un dieu-père garantissant l'initiation du dieu-enfant].

TURKELTAUB Daniel, « Perceiving Iliadic gods », HSPh 103 (2007), p. 51-81 [vigoureux plaidoyer pour une interprétation religieuse de la manière dont les figures épiques perçoivent les dieux : le poète ne fait pas de distinction entre les dieux de la machinerie divine de son poème et les dieux qui font l'objet d'un culte; il joue sur les sentiments religieux de l'auditoire dans son art poétique].

VALAVANis Panos, "Thoughts on the historical origins of the Olympic games and the cult of Pelops in Olympia ", Nikephoros 19 (2006), p. 137-152 [le fait que le vainqueur de la course allumait la flamme de l'autel de Zeus fait penser que ce concours est aussi ancien que le culte lui-même; en revanche, le culte de Pélops ne remonterait pas avant la fin du VIII / déb. VII s.]. 
VANHAEGENDOREN Koen, «Travail et loisir en Grèce ancienne. À propos de la complémentarité des activités du citoyen », AncSoc 37 (2007), p. 1-35 [notamment étude des aspects religieux de l'implication du citoyen dans les activités communataires de la cité].

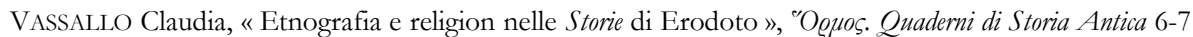
(2004-2005), p. 103-192.

VeLASCo LóPEZ María del Henar, «Ceneo, el invulnerable: su metamorfosis », Minerva 20 (2007), p. 921

VIKELA Evgenia, "Griechische Reliefweihungen an Athena. Ikonographie der Göttin und Bildkomposition der Reliefs ", $M D A I(A) 120$ (2005), p. 85-161 [l'examen des reliefs dédiés à Athéna permet d'en tracer le développement chronologique et d'en dresser des catégories, qui sont importantes pour interpréter l'iconographie d'Athéna, mais aussi la représentation que s'en donnaient les dédicants].

Villanueva Puig Marie-Christine, «Dix ans d'iconographie dionysiaque dans le monde grec », Pallas 75 (2007), p. 197-205.

VINCENT Jean-Christophe, «Recherches sur la personnalité du dieu Poséidon I. Poséidon Hippios à Mantinée et la naissance de la rivière Boyne », Gerión 25 (2007), p. 149-162 [la légende de l'aveuglement d'Aipytos renvoie au mythe i.-e. du «feu dans l'eau » qui sanctionne les impies; cet héritage invite à repenser le lien entre Poséidon et la royauté en Grèce].

VlassopOUlOU Christina, «Neue Skulpturenfragmente von der Akropolis. Beobachtungen zur Ikonographie der Athena in klassischer Zeit », $\operatorname{MDAI}(A) 120$ (2005), p. 173-210.

WeBER Martha, «Die Kultbilder der Aphrodite Urania », MDAI(A) 121 (2006), p. 165-223 [mise au point sur les types statuaires de l'Aphrodite athénienne dans la $2^{\mathrm{e}}$ moitié du Ve s. av. J.-C.; le succès de la déesse serait à mettre en relation avec la loi de Périclès sur la double ascendance des citoyens].

WHITTAKER Helène, «Burnt animal sacrifice in Mycenaean cult: a review of the evidence », OAth 3132 (2006-2007), p. 183-190.

WOLKOw B.M., "The mind of a bitch: Pandora's motive and intent in the Erga», Hermes 135 (2007), p. 247-262 [si Pandora ouvre le pithos, c'est pour y voler les provisions qu'elle croit y trouver; elle est donc bien le jouet du plan de Zeus].

ZWIERLEIN-DieHL Erika, «Les intailles magiques », Pallas 75 (2007), p. 249-262 [chronique bibliographique]. 